\title{
ON SOME DIFFERENTIAL OPERATORS FOR CERTAIN CLASSES OF ANALYTIC FUNCTIONS
}

\author{
KHALIDA INAYAT NOOR
}

Abstract. Let $f(z)=D(F(z))$ where $D$ is a differential operator defined separately in every result. Let $F$ be analytic and $F(0)=0, F^{\prime}(0)=1$. We shall find out the disc in which operator $D$ transforms some classes of analytic functions into the same.

Mathematics subject classification (2000): 30C45, 30C50.

Key words and phrases: Convex, starlike, radius, differential operator, functions with positive real part, functions of bounded boundary rotation.

\section{REFERENCES}

[1] S. D. BERNARDI, New distortion theorems for functions of positive real part and applications to the partial sums of univalent convex functions, Proc. Amer. Math. Soc., 45(1974), 113-118.

[2] W. M. CauseY AND W. L. White, Starlikeness of certain functions with integral representations, J. Math. Anal. Appl. 64(1978), 458-466.

[3] A. W. Goodman, Univalent Functions, Vol. I, II, Polygonal Publishing House, Washington, NJ, 1983.

[4] A. E. Livingston, On the radius of univalence of certain analytic functions, Proc. Amer. Math. Soc., 17(1966), 352-357.

[5] T. H. MACGREGOR, The radius of univalence of certain analytic functions, Proc. Amer. Math. Soc., 14(1963), 514-520.

[6] B. PINCHUK, Functions with bounded boundary rotation, Isr. J. Math., 10(1971), 7-16.

[7] S. RuscheweYh AND V. Singh, On certain extremal problems for functions with positive real part, Proc. Amer. Math. Soc., 61(1976), 329-334.

[8] G. M. SHAH, On the univalence of some analytic functions, Pacific J. Math., 43(1972), 239-250. 